\title{
Putting the Lab in the Lab Book: Supporting Coordination in Large, Multi-site Research
}

\author{
Francois Roubert \\ BIS, University of Westminster \\ 115 New Cavendish St, London, W1W 6UW, UK \\ F.Roubert@westminster.ac.uk
}

\author{
Mark Perry \\ DISC, Brunel University \\ Uxbridge, UB8 3PH, UK \\ mark.perry@brunel.ac.uk
}

\begin{abstract}
Large and distributed science projects present researchers with a challenging environment for interaction and collaboration. While digital technologies offer promises in supporting these difficulties, researchers appear reluctant to discontinue their use of analogue resources. We present a study of communication practices in very large-scale collaborative scientific research programmes that involve multidisciplinary and multinational research consortia. Qualitative data collection with researchers, principal investigators and project coordinators were carried out to examine the conduct and coordination of biological, biomedical and chemistry experiments that were distributed over multiple geographical locations. Results show that many problems in collaboration appear to result from the collective documentation of experimental operating procedures, tracking of experimental samples, and the sharing and cross-association of physical and digital experimental materials. Our analysis highlights the crucial but problematic role of the laboratory notebook as a driver for collaboration, most notably in supporting traceability of the distributed experimental process. We identify opportunities for improving experimental coordination, scientific communication and project synchronisation, drawing implications for digital interaction design that offers opportunities to enhance research coordination.
\end{abstract}

Collaboration, communication, distributed interaction, scientific research, scientific practice, experimental coordination, laboratory notebook, artefact design.

\section{INTRODUCTION}

The recording and sharing of scientific data to support the work of scientists involved in research has been of great interest to the Human-Computer Interaction $(\mathrm{HCl})$, Computer Supported Cooperative Work (CSCW) and Science and Technology Studies (STS) disciplines. A particular emphasis has been placed on investigating the scientific laboratory notebook (lab book), which is used by researchers directly involved in experimental work to document all the information relevant to carry out experimental procedures. In essence, the lab book, which is traditionally in paper format, is used by experimenters to keep a record of everything that takes place in the laboratory, to register and analyse intermediate experimental results, to document thoughts for future experiments, to log errors and to ensure scientific probity and experimental reproduction (Klokmose and Zander, 2010).

Because of the inherent limitations of the traditional paper format, there has been a well-documented drive for the utilisation of digital technologies to support and extend the functionality offered by lab books. Attempts at designing, developing and sometimes deploying electronic lab books have generally adopted one of these strategies: replication, supplementation, replacement or augmentation of existing paper-based lab books (Schraefel et al., 2004). Yet the overall picture of the utilisation of these new digital platforms is that the extent of the adoption and use of these tools by scientists carrying out experiments in the labs is low (Klokmose and Zander, 2010). We posit that a possible reason for this may be that the introduction of such systems is driven by research managers who are particularly concerned with standardising research practices with a view of improving monitoring, transparency and efficiency. We contend that their design is often oriented to centralised platforms that primarily support the precise logging of scientific facts and the direct accurate reporting to managers and that as such they may not be always embraced by experimenters on the ground. It appears that there is a parallel with the adoption of groupware systems that are mandated by management, in which adoption among employees is less successful (Grudin and Palen, 1995; Palen and Grudin, 2002). 
We suggest that the design of the lab book needs to shift from emphasising exclusively on off-loading (material distribution) to also focussing on shared cognition (social distribution) (Salomon, 1993). That is, we posit that the lab book needs to be completely rethought and redefined as a vehicle for research collaboration and particularly as a central platform to enable the coordination of distributed research experimental work. Particularly since the context within which scientific research takes place has changed with the emergence of research globalisation (Hoekman et al., 2010). The predominant setup for scientific research is now one of large scale multi-disciplinary projects that involve scientists operating in many institutions, sometimes spanning numerous geographical locations. Researchers in this context often need to undertake geographically distributed experimental work to take full advantage of various expertises, pieces of equipment and experimental materials at different institutions, and need to tightly coordinate their activities to maximise the impact of the experimental processes they are involved in.

This paper thus aims at exploring the role of the lab book as a vehicle for the coordination of experimental activities within large-scale distributed research projects. The following section will discuss the challenges of distributed research and review attempts which have been made at using digital technologies to mitigate these challenges through the use of digitally exteded lab books. We then describe the study we conducted to investigate the role of the lab book in supporting the coordination of experimental work and present our findings, the main themes of which are informed by the theoretical work of Salomon (1993) on distributed cognition (Rajkomar and Blandford, 2011). From these findings we derive an analysis of recommendations for the design of collaborative lab books to support experimental coordination.

\section{RELATED WORK}

Scientific research work is increasingly conducted within a distributed collaborative context and particularly as part of large multi-disciplinary projects involving heterogeneous working teams which are specialised in different research areas and which are geographically dispersed across various institutions. The scientists in these teams find themselves in positions where they need to rethink how to conduct their experimental work globally in collaboration with other partners to obtain valuable results for the project. The following section explores the impact that the rise of these distributed collaborative projects have on scientific experimental practices and the challenges that they pose to the scientists who work in them. The subsequent section delves into the changing forms and the evolving utilisations of the lab book to support these collaborative experimental practices in an attempt to provide a better framing of the exact role played by this artefact in the coordination of experimental processes.

\subsection{Distributed scientific research}

There is a history of examining collaborative research within geographically distributed, multidisciplinary and cross-institutional settings (Hoekman et al., 2010; Cummings and Kiesler, 2005, Jackson et al., 2011). Geographically dispersed cross-disciplinary collaborations are fertile grounds for the successful implementation of original ideas and actions for innovation in science (Cummings and Kiesler, 2005; Müller-Tomfelde et al., 2011). However, it is widely acknowledged that these types of collaborations pose major challenges as a result of the assembling of diverse backgrounds, cultures and expertises (Hoekman et al., 2010), particularly with regards to the project coordination i.e. the timely combination of different components of the project to achieve common goals (Cummings and Kiesler, 2005). These challenges are even intensified when the collaboration is cross-organisational. Principal Investigators find it difficult to synchronise all the efforts of a consortium of scientific partners who are not only geographically dispersed and come from different scientific backgrounds but also are accustomed to different ways of organising their work. As a result the case is often made for tighter coordination mechanisms driven by information technologies (IT) as it can have great potential for improving the coordination of the scientists' efforts although close monitoring of their efficiency is crucial (Cummings and Kiesler, 2005).

One particular thread of scholarly work has emphasised on distributed research within the European Union, and the tensions that emanate from such a model (Hoekman et al., 2010). In this environment, the increased ease of international exchanges and cross-border collaborations allows the scientists to locate better-suited partners further afield akin to pursue the same research interests and to greatly contribute to these. However, the impact of the cultivation of close proximity collaborations on the production of higher quality results is also well established (Olson and Olson, 2000). The practical organisation of the collaborative interactions within such pan-national and -continental projects therefore needs to find ways to resolve this contradiction (Hoekman et al., 2010). Resolving the issues around coordination of the work of the various consortium members will be essential to leverage the participation of each of the participants to the common research effort.

\subsection{Electronic laboratory notebooks}


The use of the lab book as an artefact which plays an essential role in the scientific experimental process has attracted a fair amount of attention in the research literature. The emphasis has often been placed on exploring how the lab book is embedded within the scientific practice of the researchers, often with a view to provide design specifications or/and develop digital extensions of this artefact which can help shaping the work of the experimenter in a meaningful manner. What has become apparent from this line of enquiry is that the lab book - both in its physical form and digital configuration - can have a wide range of formats, structures and utilisations in scientific projects (Mackay et al., 2002) which vary depending on the scientists' personal organisation of work and levels of expertise (Sarini et al., 2004).

For the scientists who conduct their experimental work 'at the bench', the recording of scientific data in the lab book has long been advocated as the 'correct' way of recording scientific observations and encouraged by management. The lab book is typically the place where the details and timings of the execution of experimental procedures are methodically recorded alongside the related observations, initial analyses and preliminary results (Sarini et al., 2004). Yet, the lab book is not limited to a linear description of experimental methods and techniques, and can assemble a wide range of heterogeneous materials such as aidememoires for particular techniques, pointers to external documents, printouts of images, snippets of data, partial results, spontaneous observations, or early interpretations. In practice, it is often used as a first port of call for the many iterations of the interpretation process that takes place as part of the experimental work to make sense of the results (Mackay et al., 2002). However the shared interpretation of these results by various collaborators can be complicated because of the personal styles and expertises involved (Sarini and et al., 2004).

A small number of studies have investigated the attempt made by scientific teams to use IT to assist the individual and collective recording and organising of scientific experimental information (Schraefel et al., 2004; Mackay et al., 2002; Sarini et al., 2004; Myers, 2003; Schraefel and Dix, 2009; Tabard et al., 2008). They report rather persuasively on the design and development of digital systems to give scientific teams ubiquitous platforms to be used in and out of the laboratory to support the information needs in the management of their research-based operations. A wide variety of extended functionalities is advocated to maximise the benefits of digital technologies. These include features like:

- editing of entries, searching, linking to external documents, capturing users' gestures, recommending actions (Mackay et al., 2002);

- digital signatures, protected information flows, comprehensive logging, timestamps (Myers, 2003);

- master notebook, cross- linking of temporal events to activities and reviewing of others' comments (Tabard et al., 2008);

The main emphasis of this body of work is thus mostly on the liberation from the physical constraints of the paper format and the affordances brought in by digital technologies to provide the scientists with additional information benefits such as flexibility and enhanced communications.

We posit that the prevailing focus on overcoming the constraints of the paper-based medium is somehow limited. There is a need for exploring the role of the lab book in the increasingly complex context within which the scientists are operating which is one of distributed multi-site research and within which they often have differing needs and practices. We suggest that the multiple facets of this artefact - in terms of formats, structures and utilisations - is what makes it a challenge to produce digital extensions of lab books which can satisfy everyone's needs and be truly adopted by working scientists. There is a subsequently a necessity to closely examine the way with which the lab book can be enhanced to assist the complex interactions between the various stakeholders of these distributed projects and the networked coordination of their scientific practices.

It is around this backdrop of questions and expectations that we have developed our own research programme: to examine the practices, needs and problems faced by research scientists and project leaders in doing their science across multiple teams, institutions, disciplines and sites.

\section{RESEARCH DESIGN AND METHODS}

We organised our investigation around the two following research aims: 1) to gain an insight of the complex multi-dimensional collaborative context within which scientists involved in international multi-disciplinary projects operate and set up their scientific communication; 2) to construct an indepth understanding of the role of the lab book - in connection with other experimental artefacts - as a vehicle for the scientific communication required to support the coordination of distributed experimental activities.

\subsection{Exploratory interview-based study}

The study consisted of twenty two semi-structured interviews with research collaborators with different roles and different levels of experience - past and previous - in a range of research projects in a wide 
range of scientific fields. These participants worked in research institutes across ten European countries and were all involved in large, distributed European research projects. The breakdown of the number of participants for their various roles and for the scientific fields in which they worked is presented in table 1.

Table 1: Breakdown of participants' roles and fields

\begin{tabular}{|l|l|l|}
\hline \multirow{4}{*}{ Role } & Principal Investigator, project coordinator & 5 \\
\cline { 2 - 3 } & Senior researcher, work package leader & 11 \\
\cline { 2 - 3 } & Researcher, PhD, post-doc, lab technician & 6 \\
\hline \multirow{5}{*}{ Field } & Biotechnology, biomedicine \& nanomedicine & 8 \\
\cline { 2 - 3 } & Systems biology and genomics & 4 \\
\cline { 2 - 3 } & Microbiology and cell biology & 3 \\
\cline { 2 - 3 } & Biochemistry & 2 \\
\cline { 2 - 3 } & Food science & 2 \\
\cline { 2 - 3 } & Infectious diseases & 2 \\
\cline { 2 - 3 } & Molecular biophysics & 1 \\
\hline
\end{tabular}

These semi-structured interviews were all conducted remotely using a voice-over-Internet Protocol service. The interview schedule was carefully designed to enquire on intra-consortium interactions with a particular emphasis on scientific communication practices. Interviews were all conducted between February 2012 and November 2012 and lasted between forty and seventy five minutes.

We used a thematic analysis (Boyatzis, 1998) to conduct a qualitative analysis of the collected data. The identification of high-level themes was informed by the distinction made by Salomon between two classes of distributed cognition (1993): Off-loading and Shared cognition. It was then decided that a third emerging one, Reflection, ought to be considered as it was given prominence by most participants. For each theme, sub-themes then surfaced and were used to organise our findings and the associated implications for design. A summary of the themes, sub-themes, findings and mapped implications for design is presented in table 2.

\section{FINDINGS}

A number of recommendations exist - e.g. (Ebel et al., 2004) - that call for lab books to be highlystructured paper-based repository of a scientist's activities which should be used in-situ to make a permanent and meticulous record of the experimental procedures. These cover a wide variety of heterogeneous information such as practical experimental techniques, raw thoughts and observations, research hypotheses or initial findings. Such an expectation of use appears to be an especial concern for senior researchers and members of project coordination team. "It is a requirement for our project. All the partners involved should use the same lab notebook with the same format to record everything they do" (Scientist A). However, our observations on lab book use, and the values that they hold, would appear in part to contradict this and in many instances the lab book was not restricted to this one-dimensional, highly-structured physical medium. Lab books clearly encompass different meanings for different people and different contexts as their use varied greatly across laboratories and projects. In some ways, the term 'lab book' would appear to be more like a metaphor for the sum of recorded experimental documentation, rather than the unique, personal, structured record of a researcher's activities that is alluded to in the research methods literature.

In the sections that follow, we examine the manner in which researchers describe the ways that they create, maintain, use, value and find problems with lab books in their work, evidencing this with specific reference to examples from our data.

\subsection{Logging scientific activity}

Many of the scientists and technicians reported capturing raw information relevant to the operations they were performing on a range of different media. These were typically paper-based and consisted of rough papers, sticky notes and labels, printouts (often annotated), scrapbooks, notepads, or a variety of combinations of these.

\subsubsection{Immediacy, media availability and utility}

The commonly used materials for in-situ documentation of the experimental procedures were those that were 'to hand' at the time of use, in an environment that was often poorly populated with recording materials to select from. The use of such physical materials can be easily justified by the constraints of the environment in which the experimental work often takes place. For instance, the conditions in a wet laboratory, where volatile chemicals, materials or biological matters are handled, or laboratory requirements to avoid contamination ( $\mathrm{Li}$ et al., 2012) may impact on the types of media that can be used. In many instances, the value that these materials held for users was not always immediately apparent to the researchers, yet at the same time they recognised that they might have potential value. Often, this cost-benefit dilemma was not possible to immediately resolve, and as a consequence, the information on these media failed to make the transition to a more formally recorded format. Nevertheless, these materials required archiving, often with some further limited annotation to contextualise and make sense of the circumstances of their collection. To aid recall and 
Table 2: Mapping between themes, findings and implications for design.

\begin{tabular}{|c|c|c|c|}
\hline Themes & Sub-themes & Findings on lab book use & $\begin{array}{l}\text { Implications for enhanced lab book } \\
\text { design }\end{array}$ \\
\hline \multirow{2}{*}{ Off-loading } & $\begin{array}{l}\text { Logging of scientific } \\
\text { activity }\end{array}$ & $\begin{array}{l}\text { Capturing facts in restrictive lab } \\
\text { conditions }\end{array}$ & $\begin{array}{l}\text { Lightweight mobile solution, ubiquitous } \\
\text { spoken notes, additional visual content }\end{array}$ \\
\hline & Organisation of data & $\begin{array}{l}\text { Using chronological or project- } \\
\text { based structure }\end{array}$ & $\begin{array}{l}\text { Chronological structure, accommodating } \\
\text { multiple activities, search ability, tagging }\end{array}$ \\
\hline \multirow{2}{*}{ Reflection } & Review of content & $\begin{array}{l}\text { Editing \& refining content and } \\
\text { connecting to other sources }\end{array}$ & $\begin{array}{l}\text { Multiple edits, keeping track of timings of } \\
\text { edits, linking up with previous entries }\end{array}$ \\
\hline & Reflective interpretation & $\begin{array}{l}\text { Restructuring content for sense- } \\
\text { making \& permanent archiving }\end{array}$ & $\begin{array}{l}\text { Reflective commentaries, contextualising } \\
\text { content, archiving of multiple versions }\end{array}$ \\
\hline \multirow{2}{*}{$\begin{array}{l}\text { Shared } \\
\text { Cognition }\end{array}$} & $\begin{array}{l}\text { Experimental and } \\
\text { operational coordination }\end{array}$ & $\begin{array}{l}\text { Collective protocol-driven } \\
\text { experimental development }\end{array}$ & $\begin{array}{l}\text { Linking entries with multiple experiment } \\
\text { attempts and multiple protocol iterations }\end{array}$ \\
\hline & $\begin{array}{l}\text { Coordination of material } \\
\text { exchange }\end{array}$ & $\begin{array}{l}\text { Ad hoc or planned exchange of } \\
\text { physical and digital materials }\end{array}$ & $\begin{array}{l}\text { Linking entries with sample logbook, } \\
\text { subscription to a sample, timestamp }\end{array}$ \\
\hline
\end{tabular}

reuse they would need to be stored with other content collected at the same time, some of which might be in different medium. This set of mixed media would naturally lead to difficulties in indexing and cataloguing content, not all of which could easily be formed into the common format of lab notebook referenced by Scientist $A$ above.

Despite these difficulties in recording information, the structure of recorded material would appear to carry importance with many of the researchers interviewed. When the choice of lab book format was not imposed, some researchers themselves chose to use highly-structured approaches, such as permanently bound notebooks with numbered pages. "I have this notebook in which I record everything that is happening. For every assay or test I create a new entry and I write down the date and time. Then I take a note of all the things that I do, of the problems that I have and the thoughts that come to me as I am doing the work; everything that I think may be useful later" (Scientist B). What is interesting in this structured approach is not that she has developed a formal structure, but that this structure is generalisable across topics and media, and can be referenced like a scientific diary in which the activities that are performed are systematically entered as the experiment is progressing. This thus provides a chronological and quasi-permanent record of the immediate observations and insights made by the researcher in action, but which can accommodate different types of observation or content.

However, the constraints of even such an open approach to structuring content could also be perceived as too difficult to manage by other researchers. "I used to keep one main structured lab book but things became too complicated as I am doing too many things for too many people" (Scientist C). Such a comment about the practical problems in recording lab notes for activities carried out quasi-concurrently across multiple research projects is revealing about the difficulties faced in making a single lab record. Scientists that carry out many different activities may find it restricting to adopt a rigid time-based organisational strategy to document their activities. This could be particularly the case for researchers involved in one or more large distributed scientific projects that run in parallel and for which they may have to perform often unrelated experimental tasks. "I want to keep together the things that are relevant to each other. So I write things on sheets of paper and I file them together for everything concerning a specific part of the project" (Scientist D). So it is clear that for her, recordings lab activities are still important, and that some form of categorisation or structuring is considered useful, but that a single record of her lab-based activities carries little relevance for her work, and organising her observations and activities in a project-based or thematic manner allows for more a practically useful approach.

\subsubsection{Reflecting about the raw facts}

Following the raw capture of experimental thoughts, many of the scientists appeared to undertake a refining process to make sense of the disparate elements which were recorded during the note-taking exercise while conducting the experiment. "After the experiment, I sit at the computer and go through my notes. I think about how I did things and I write about it in an organised way on the computer" (Scientist E). These more reflective interpretations were often described as being performed on personal computers and would then be digitally archived following carefully considered file organisation systems to enable timely retrieval and efficient re-use. "I have this Project folder and there a folder for Experiments and one for Simulations. Inside each of these there is another one called Tasks where I write down information about the different things that I do in an experiment or a simulation" (Scientist B). What seems apparent is that maintenance of the (now digital extended) lab book allows participants to keep a dynamic and evolving record of the practice of their experimental work, to organise and structure it in a customised manner which can fulfil their specific needs and to build a digital archive 
which is highly searchable and manageable. However this practice of digitally-extended reflection on lab activities can create problems with data fragmentation and long-term archiving.

Recording of the operational details on multiple platforms, physical and digital or a mixture of both may result in problems for recall. "Sometimes my notes get a bit messy. I have bits of information all over the place, on sheets of papers, sticky notes and text files on various computers" (Scientist $\mathrm{C}$ ). The researcher ends up collecting snippets of unrelated items that are difficult to retrieve and to use. The relationship between the physical information captured on a variety of heterogeneous platforms and the more reflective information recorded digitally was reported as somehow confused and the traceability from the digital entry to the original source appeared to be problematic. This appeared to be an issue particularly at the reflective stage as laboratory notes which needed to be compiled and organised in a meaningful manner appeared to be difficult to locate and completely de-contextualised and hence difficult to make sense of.

\subsection{Coordination of the experimental process}

In the context of a research project, we repeatedly observed how the lab book often served a more complex purpose than just logging experimental activities for a scientist's personal use. In projects where the experimental work that needs to be undertaken is distributed across cross-institutional and cross-disciplinary research teams, the lab book appeared to play a central role in the organisation and coordination of the collaborative experimental work. At its simplest level this may simply be a comparison of data from the lab books. "When we do an experiment in the lab or across several labs, we have to use a protocol. We sometimes call it a SOP, a Standard Operating Procedure. We try to follow it step by step and we take notes in our lab books as we are doing it, then we compare our notes and we discuss it" (Scientist F). This flexible ability of the lab book to note and record ad hoc content meant that it was used as a central vehicle for the coordination of complex collaborative enquiries. In this respect, the role of the lab book that is described by Scientist $F$ shows its close relationship with a key research artefact essential to the coordinating the investigatory work undertaken: the research protocol. The following section explores how the close interplay between the lab book and the research protocol supports the designing, conducting, coordinating and documenting of experiments within a distributed research context.

\subsubsection{Protocols and experimental coordination}

We found that scientists working as part of a distributed project would be conducting usually multiple experiments to solve a particular problem or test a particular hypothesis. The same scientist would undertake many experiments in their lab to ensure that the experiment is repeatable locally or would physically go to a different site to work alongside their partner and ensure that the experiment was repeatable remotely. Other scientists may also attempt to recreate the similar experiment either in the same lab for local reproducibility or at a different location to validate distributed reproducibility. The multiple experiments on a particular line of enquiry could be thus divided in 4 main types of cycles as summarised in table 3 .

Table 3: Experimental cycles in distributed project

\begin{tabular}{|l|l|}
\hline - Same lab \& same scientist & local \\
- Same material \& same apparatus & repeatability \\
\hline - Same lab \& different scientist & local \\
- Same material \& same apparatus & reproducibility \\
\hline - Different lab \& same scientist & distributed \\
- Same material \& different apparatus & repeatability \\
\hline - Different lab \& different scientist & distributed \\
- Same material \& different apparatus & reproducibility \\
\hline
\end{tabular}

All of these experimental cycles would be based on one or more protocols which, for each iteration, would get referenced in the lab book. When designing a new experiment, the scientist would usually enquire with fellow researchers whether they were familiar with a particular set up or would happen to have a similar protocol. The fellow scientists would consult their own lab books and usually send the original experimenter either a full protocol or a description of particular techniques or dosages. The experiment designer would then adapt the protocol or use the techniques to create a new protocol to suit their needs. " $A$ protocol is a bit like a cooking recipe. You don't always have all the ingredients or the tools that you need. So you need to be creative about it, make it work for you" (Scientist G). This process of designing a new experiment also often involved the support of other team members or lab technicians, resulting in the collective generation of a new protocol: "When you design your new experiment you have to try things in the lab, like techniques that you know or that your colleagues know. And you write down your attempts in your lab book. And then eventually at the end you manage to write a new protocol" (Scientist H).

This setting up of a new experimental protocol thus appeared to be a highly iterative process during which the researchers involved in this task collectively adopted a trial-and-error approach. This would then be closely documented in the scientists' lab books: "We get the protocol we need and it gives us the general direction for our experiment. Then we do our own thing and we try to log everything. At the end I sit down and write a new 
version of the protocol, one that suits our needs, and I keep it in the lab folder so that we can all get back to it later on" (Scientist G). The development of the protocol alongside the multiple attempts to ensure repeatability and reproducibility would be carefully logged in the lab book for later use in experimental design, internal reporting, ethical clearance, publication or training.

\subsection{Managing the exchange of experimental material}

An important aspect in a distributed scientific project like the ones described above involves the controlled exchange of experimental samples between labs: "They produce the particles to suit our needs and then they send them to us. We can then use them for our tests" (Scientist I). In our observations, the lab book in its general sense - a log of experimental activities - also played an important role in exchanging experimental samples because of its close relationship with another artefact used to manage this process: the sample tracking logbook. The following sections explore the how the interchanges between this (collectively used) artefact and the lab book influences the coordination of the complex exchanges of materials which need to take place between the various research partners to allow experiments to be conducted as part of the distributed project.

\subsubsection{Ad hoc material exchange}

The scientists reported many instances where samples had to be sent between sites. This would range from chemical substances, batches of particles, organs or entire animals. Often these exchanges were ad hoc and did not require any particular pre-planning. Scientist $J$ for example, works on a large European multi-disciplinary project and has to closely collaborate with half a dozen partners located on four different sites. "Sometimes as part of my experiment I want to try something and for that I need them to send me a sample. So I just send them an email explaining what I am after and ask if they can send it to me" (Scientist $\mathrm{J}$ ). The researcher in need of the sample for designing an experiment would refer to their lab book to produce a list of requirements for particular materials. The researcher who would receive the request would then post the required materials and typically send a confirmation email. Sometimes a physical document would be enclosed in the package alongside the samples to provide additional information such as the characterisation of the sample or the conditions of storage and use. This information would typically be taken from their own lab book. On occasion, this would be more sophisticated, and where the sample required a special treatment regime during transit, a digital probe might be included in shipments, such as a digital USB-powered thermometer and hygrometer to keep a track of the temperature and moisture levels of the contained material.

In some instances, the researcher organising the shipment also sent, spontaneously or upon request, additional information on the posted samples in an electronic format, typically in an email, particularly if this was key to the experimental work conducted at the other end. It appeared that in certain cases, the dissemination of heterogeneous digital and physical information associated with the exchange of physical goods could be quite problematic: "Sometimes it gets all a bit messy. I have to check the email I sent explaining what I was after, the sheet they posted with the sample and the email they sent me as well. And sometimes it is not the same. Particularly if the sample is not exactly what I wanted and I have to ask for another one. It all gets a bit confusing" (Scientist K). This relationship between physical and electronic information about the samples gives an insight into the nature of the experimental process. It is not simply that these experimental materials are things that can be used in the laboratory, but that they carry additional invisible layers of meaning that impact on their use - layers that will determine their use and the interpretations that can be made from their use in experimental conditions. Cross-referencing information on these samples is understandably complex, and recording mixed media content over digital records and across various peoples' lab books can prove practically difficult to coordinate.

\subsubsection{Tight coordination of sample exchange}

This issue of locating and assembling heterogeneous physical material and digital resources caused particularly problems when experiments needed to be performed under closely controlled conditions as a requirement of the nature of the scientific work done in the project. Time constraints sometimes came into consideration if the exchanged material would have a short lifespan or if they were at risk of changing state. Such issues of exactness are critical in controlled experimental design, and a complex networked exchange of samples usually has had to be methodically synchronised in order for the distributed experimental work to be successful: "We have this testing plan that we have agreed in the work package. By this date we need to do this experiment. For this we need a certain type of particles with a certain type of coating. So we send them a reminder and the exact descriptions of the particles we need. Or if we are late, we let them know that we will need them later. Then they send them to us and to the other partners who are doing similar work. We do our tests separately and then we talk" (Scientist L). In order to ensure that the exchanges are carried out as planned, documentation usually has to be disseminated 
between the various partners involved to provide them with the information required for them to set up their experiments. These could take many forms, such as emails, written notes or even sometimes, verbal data transmitted over the telephone. As before, this mix of materials was supported by the use of the lab book to provide the required information (sender) and to record this information as part of the experimental set-up (receiver). As we observed, these multiple complex exchanges of physical material and associated physical and digital artefacts were not always organised in a structured manner and the dispersion of information over different physical and digital platforms could lead to some confusion and the potential for error because of the distributed project participants.

\section{DISCUSSION AND DESIGN IMPLICATIONS}

The findings highlight the complexities related to the recording and sharing of experimental data within the context of large multi-site distributed experimental research projects. We posit that the attempts previously made to design and implement electronic lab books to benefit from the affordances offered by IT have somehow fallen short of capturing these complexities and particularly have overlooked the complex interplay between the lab book and the other essential artefacts used at the centre of the coordinated experimental process i.e. the experimental protocol and the experimental sample logbook. However, we are fully aware that there is a trade-off between the high volumes of functionalities that an electronic lab book can offer and its flexibility and ease of use, and hence its adoption by scientists. It is thus very challenging to capture all the complex aspects of the work of the experimenters discussed earlier within one system and to design a system that can support the full range of their working practices in a simple manner. This issue is amplified by the high variety of scientific practices present in a large multidisciplinary and multi-site project, which involves a wide range of scientific practitioners with different information requirements and different ways of managing their scientific data.

We contend that in the context of multi-disciplinary and cross-institutional research which is increasingly predominant, that the lab book needs to be rethought as a central vehicle for collaboration with an emphasis on the coordination of distributed experimental processes. As such, and in the lights of our findings, we identify opportunities to support the utilisation of this artefact more effectively in this context and in relation to other artefacts, and offer design implications for future systems. These are summarised in table 2 presented earlier.

\subsection{In- and ex-situ use}

The findings show a tension between the demands on user's attention and time for immediate use of the lab book in a 'lightweight' design solution, and the follow-on uses to which recorded material may be put in reflection at a later time (by the content creator, or by other users), in which lab notes in lightweight format may carry insufficient details to be put to good use. Nevertheless, a technology solution that requires complex interactions and high levels of detailed content completion appears unlikely to be used effectively, because of the overheads this would place on lab workers - see (Schraefel et al., 2004). This overhead is likely to be exacerbated because of the complex conditions that many experiential studies are carried out in, such as the introduction or release of contaminants as material moves between the lab and off-lab sites. Where the cost to the users is high, but the benefits that they see as individual may be low, there is an additional burden for users in creating and managing content, leading to a potential for under-reporting, something that seems to fly in the face of much of the curiosity-driven documentation currently performed in the lab book. One technical solution that might aid lightweight content creation in limiting lab conditions could be for instance the use of ubiquitous technologies enabled with a headphone/ microphone adapter for taking spoken notes which could be used easily as a hand-free device while conducting experiments. The essential chronological aspect of current lab book practice would be - and ought to be - maintained: entries are in almost all instances ordered by the time they occur, allowing simple search by time, and sequential activity orderings to be easily determined. This, in combination with photographic images (or experimental set-ups, equipment screens and printouts, sketched notes) alongside possibly voice recognition might provide ways of adding in-situ richness to digital records in a way that form-based computer lab book entries (as seen in many current systems) cannot. Such enriched logbook recordings would allow different teams who are conducting experimental tasks in parallel to immediately record and exchange information crucial to the experimental work. Doing this on a mobile device is likely to offer advantages in moving away from one of the problems that we observe in the fragmentation of data over multiple sources.

While chronologically ordered records provide an opportunity for lightweight recording and their subsequent search, the participants also note that they sometimes need to search by different parameters, for example, by project or work package, and systems may need to be flexible enough to accommodate differing operational practices by users, some of whom may wish to 
record and share content between several projects, or indeed over multiple sites, or organisations. Some form of simple 'tagging' may be helpful here (that may be carried out in-lab), providing a loweffort way of categorising content that is of relevance to particular groups or which relates to particular topics (e.g. an observation or protocol).

We have seen that reflection away from the 'bench' is an important time that lab book content may be reviewed and 'editorial' or reflective commentaries added, sometimes for the benefit of other readers of the lab book (for collaboration with colleagues or progress monitoring by lab managers). Any digital redesign of the lab book should consider allowing users to restructure content, and to add commentaries that may be shared and meaningfully used by others them. One of the advantages over paper-based formats of digital content is that both the original and edited versions can co-exist; moreover, the chronological features of when edits are made to content may also be of value in reviewing the lab book and making sense of these edits. Of course, visualising how these multiple views into the data are to be presented to its various users remains a non-trivial interaction design task.

\subsection{Intra-team and multi-site experimental coordination}

One of the core concerns for scientists in their interaction between team members and across sites lies in showing how the work was done, and is linked to one of the highest aspirations of science itself: experimental reproducibility. In this respect, a coordination-driven lab book needs first and foremost to establish a clear link with the other essential artefact in use: the experimental protocol. As we have seen, experimental design is an iterative process that relies on the interplay between: a) standardised, existing protocols of experimental techniques, material and equipment; b) individual scientists' lab books or notes that form a record of the practical conduct of the experiment i.e. material used, equipment used, set-up, calibrations, techniques, dosages, etc.; and c) scientists' prior experience and tacit know-how that may be communicated across different media. A redesigned digital lab book offers an opportunity to support this interplay. One way that this could be achieved is by providing links between lab book entries by different people (at inter-team, intra-team or intra-lab levels) for the various experiments and protocols developed.

\subsection{Tight and loose coordination of material exchange}

As we have seen in the data, experimental reproducibility also often requires that the physical samples that are used in tests be controlled in some way (temperature, purity, etc.) or calibrated to the same conditions as in other studies at other sites. At the core of this, we also see the logbook being used in managing how these details are recorded and used. It would therefore be pertinent to establish links between the lab book and the experimental sample logbook. This control over the exchange of material is made more pertinent when it is considered that it is not just the material exchanged that is critical, but there is often a set of requirements from the lab requesting the sample that need to be recorded, and a set of handover details that accompany the sample that will include details on how it has been packaged and transported, any unique characteristics, and how it is to be stored or used. The variety of media that accompany such exchanges (from emails, paperwork, barcodes, postmarks or receipt confirmations, databases or spreadsheets, and physical items, such as thermometers and USB devices) makes for a highly fragmented array of items to bring into coordination and collate together into a record of the sample. The nature of this exchange is often ad hoc, and reifying this process into an 'optimised' formal technology record therefore seems an unsuitable solution. The chronological nature of these exchanges, may however offer an opportunity for design: these tend to develop along a temporal dimension. As we have seen, information about the sample exchanges are often recorded in a 'sample logbook' that is held in the lab. Currently these may be held in analogue (or mixed media) form, or in a digital format (on a database or as documents on a shared drive). One solution to connecting a digital lab book and a digitally held logbook of samples may lie in allowing lab book users to 'subscribe' to a sample in a digital record (connecting to a database record, or simply to a file or folder on a hard drive), that would 'stamp' a record of any changes to it into their digital lab book, and raise an alert to its use of this change. This would allow users to be made aware of any sample characteristics developments, and that this be entered into the lab book in the same chronological way as any other experimental observations that they made themselves.

\section{CONCLUSIONS}

This study examined the role of the lab book as a collaborative artefact, used not only to record experimental information but also to share it, and this within the context of large multi-site and multidisciplinary scientific projects. We argue that to enable potentially valuable digital extensions of lab books to be fully accepted by the scientists who conduct experiments, as opposed to be seen as mandated by research leaders, the role of the lab book needs to be redefined and that this artefact 
needs to be placed at the centre of the coordination of distributed experimental processes. For this repositioning to be beneficial to the experimenter, we make a case for an extended digital design that provides a lightweight chronological recording of 'live' experimental data combined with other rich ways of capturing essential information as the experimental work is being carried out in synchronisation between various sites. A tagging feature could also be introduced to provide the necessary flexibility to point at the information relevant to various groups or work packages. For complex networked experimental work, we contend that coordination-driven lab book entries should be linked up to relevant standardised experimental protocols and their utilisation/editing should be interconnected with the tracking of related experimental materials to enable the temporal exchange of samples and accompanying digital and physical information and thus to allow the effective synchronisation of reproducible experimental activities. We contend that these recommendations towards the design of a networked ecology of digital and physical research artefacts have the potential to be applicable to a wide range of distributed cross-institutional and multidisciplinary scientific projects.

\section{REFERENCES}

Boyatzis, R.E. (1998). Transforming qualitative information: Thematic analysis and code development, Sage Publications, Thousand Oaks, USA.

Cummings, J. and Kiesler, S. (2005). Collaborative research across disciplinary and organizational boundaries. Social Studies of Science, 35(5), 703722.

Ebel, H. F., Bliefert, C. and Russey, W.E.(2004): The art of scientific writing: from student reports to professional publications in chemistry and related fields, 2nd ed., Wiley, Indianapolis.

Grudin, J. and Palen, L. (1995). Why groupware succeeds: Discretion or mandate? In Proceedings of ECSCW'95, pp. 263-276, Springer-Verlag, Berlin.

Hoekman, J., Frenken, K. and Tijssen, R. J. W. (2010). Research collaboration at a distance: Changing spatial patterns of scientific collaboration within Europe. Research Policy, 39(5), 662-673.

Jackson, S., Ribes, D., Buyuktur, A. G. and Bowker, G. C. B. (2011). Collaborative rhythm: temporal dissonance and alignment in collaborative scientific work. In Proceedings of CSCW 2011, pp. 245-254. ACM, New York.
Klokmose, C. N., and Zander, P. (2010). Rethinking Laboratory Notebooks. Proceedings of DISC 2010, pp. 119-140. Springer London.

Li, J., Robertson, T., and Muller-Tomfelde, C. (2012). Distributed scientific group collaboration across biocontainment barriers. In Proceedings of CSCW 2012, pp. 1247-1256, ACM, New York

Mackay, W., Pothier, G., Letondal, C., Bøegh, K. and Sørensen, H. E. S. (2002). The missing link: augmenting biology laboratory notebooks. Proceedings of UIST '02, pp. 41-50, ACM, New York.

Müller-Tomfelde, C., Li, J., and Hyatt, A. (2011). An integrated communication and collaboration platform for distributed scientific workgroups. In Proceedings of Interact'11, LNCS, pp. 248-258, Springer-Verlag, Berlin.

Myers, J. (2003). Collaborative electronic notebooks as electronic records: Design issues for the secure electronic laboratory notebook (ELN). In Proceedings of CTS'03, IEEE, New York.

Olson, G., and Olson, J. (2000). Distance matters. Human-computer interaction, 15(2), 139-178.

Palen, L., and Grudin, J. (2002). Discretionary adoption of group support software: lessons from calendar applications. In Munkvold, B.E. (ed.), Implementing collaboration technologies in industry, Springer-Verlag, Berlin.

Rajkomar, A., and Blandford, A. (2011). Distributed cognition for evaluating healthcare technology. Proceedings of BCS HCl 2011, pp. 341-350.

Salomon, G. (1993). No distribution without individuals' cognition: A dynamic interactional view. In Salomon, G. (ed.), Distributed Cognitions, Cambridge University Press, New York.

Sarini, M., Blanzieri, E., Giorgini, P. and Mozer, C. (2004). From actions to suggestions: supporting the work of biologists through laboratory notebooks. In Proceedings of COOP 2004, pp. 131-146.

Schraefel, M. C., and Dix, A. (2009). Within bounds and between domains: reflecting on Making Tea within the context of design elicitation methods. International Journal of Human-Computer Studies, 67(4), 313-323.

Schraefel, M. C., Hughes, G., Mills, H., Smith, G., Payne, T. R. and Frey, J. (2004). Breaking the book: translating the chemistry lab book into a pervasive computing lab environment. Proceedings of CHI '04, pp. 25-32. ACM, New York.

Tabard, A., Mackay, W. and Eastmond, E. (2008). From individual to collaborative: the evolution of prism, a hybrid laboratory notebook. Proceedings of CSCW '08, pp. 569-578. ACM, New York. 